\title{
Esthetic preference for rectangles of vertical and horizontal orientation
}

\author{
ROBERT W. KUBEY and ALAN L. BARNETT \\ Rutgers University, New Brunswick, New Jersey
}

\section{(H. R. Schiffman, Sponsor)}

\begin{abstract}
Esthetic preference for vertical and horizontal rectangles was tested. The study was stimulated by renewed interest in television screen aspect ratios. Previous work by Schiffman $(1966,1969$, 1978) on rectangle orientation had resulted in contradictory findings. In the present study, pairs of slides of identical solid-colored rectangles that varied only in horizontal or vertical orientation were embedded in a larger protocol of similar slide pairs and presented to 183 male and female undergraduate volunteers. Other slide stimuli were also presented and tested for preference and self-reports of relaxation upon viewing. The findings demonstrate no evidence of any predisposition toward a preference for one orientation over the other.
\end{abstract}

In the early 1980 s, engineers and scientists at major television manufacturing companies, such as Sony and General Electric, began experimenting with different television-screen aspect ratios in an effort to determine the ideal proportion for future design. With the coming of high-definition TV (HDTV), television manufacturers concluded that they needed to resolve the problem of optimal screen proportion. Previous research on the "golden proportion"' (or section), so prevalent in Greek architecture, wherein the smaller side of a rectangle is 0.618 times the larger, was of particular interest in these industry studies. The traditional television set with which we are familiar has a short-to-long side ratio of three-to-four, or 0.750. Japanese companies pioneering HDTV have generally settled on a more horizontal screen, approaching the aspect ratio of Cinemascope, with a short-to-long ratio of three-to-five-and-one-third, or 0.562 .

In point of fact, and contrary to popular legend, the golden proportion has been generally shown to result in no special psychological response (Hintz \& Nelson, 1970; Plug, 1980; Schiffman, 1966, 1969; Schiffman \& Bobko, 1978), although it is true that proportions close in ratio to the golden proportion do seem to be generally preferred to more square-like presentations.

Television engineers told us they were concerned with determining the optimal proportion only for television screens oriented horizontally (horizontally dominant) with respect to the long side, coincident with the orientation of the binocular visual field. Krugman (personal communication, March 7, 1986), however, reports that a vertically dominant television screen is more pleasing. This could be attributed to a relationship between relaxation states and patterns of eye movement, or conversely to the coincidence of left-brain activity and pleasure with gross or more vertical eye movements (Krugman, 1971; Mul-

The authors' mailing address is: Department of Communication, Rutgers University, 4 Huntington Street, New Brunswick, NJ 08903. holland, 1974), or to a variety of other factors. Literature in the study of hypnosis and other altered states of consciousness suggests that vertical eye movement could also be related to states of deep relaxation.

In the study reported here, we sought to determine whether there was a visual preference for horizontal or vertical orientation of a rectangle and whether self-reports of relaxation varied with different orientations of rectangles. Only Schiffman (1966) had previously tested for this preference, and he concluded, in one study, that there was a significant preference for rectangles oriented horizontally with respect to the long side. Schiffman's study required subjects to draw a single, esthetically pleasing rectangle and then to indicate preferred horizontal/ vertical orientation.

In a second study, Schiffman (1969) found a slight, but nonsignificant, preference for rectangles oriented with the long side vertical. Stimuli (cards in one experiment, slides in another) were presented $1 \mathrm{~m}$ from the subject. In the first experiment, the long side of each card measured $12.70 \mathrm{~cm}$ and the short side varied so that the ratios of the short to the long dimensions were $0.318,0.418,0.518$, $0.618,0.718$, and 0.818 . In the second experiment, with the exception of 0.818 , the same ratios were used and the projected images were no larger than $56 \times 38 \mathrm{~cm}$.

The question of whether or not there was a distinct preference for horizontal or vertical orientation in rectangles remained unresolved. Furthermore, Schiffman's studies employed very few subjects (36 undergraduates in the first study) and only males in the second study ( 25 in the first experiment and 20 in the second). The present study differed in a number of respects.

\section{METHOD}

\section{Subjects}

The subjects were 183 undergraduate students ( 98 male, 85 female) enrolled in an introductory psychology course. They were unaware of the experiment's purpose. 


\section{Stimuli}

Stimuli for Part 1 were 28 pairs of slides consisting of rectangles (with aspect ratios of 0.452 and 0.548 ) of three different colors (blue, red, and green), other geometric shapes of the same colors, and color photographs of actual scenes and still lifes (aspect ratio 0.676) presented either horizontally or vertically.

Each presentation consisted of a pair of same-size vertical and/or horizontal rectangles, geometric shapes, or vertical and/or horizontal photographs. The test stimuli, embedded within the larger protocol, were the 10 pairs consisting of a vertical and horizontal rectangle of the same color (e.g., a red horizontal rectangle paired with a same-size red vertical rectangle) or a vertical and horizontal photograph (a horizontal stilllife photograph paired with a vertical photograph of the same still life).

Test stimuli were randomly distributed throughout the screening, and in such a manner that six of the pairings presented the horizontal rectangle on the left and six pairings presented it on the right. Upon debriefing, no subjects were able to pinpoint the test stimuli.

Other nontest combinations included geometric shapes paired with rectangles of the same or different colors, paired rectangles of different colors but the same orientation, and pairs of dissimilar photographs paired with each other or with other geometric shapes.

Stimuli for Part 2 consisted of 14 single slides of rectangles, other geometric shapes, and photographs. The test stimuli embedded within these slides were one pair of separately projected identical, same-colored horizontal and vertical rectangles and one pair of separately projected identical horizontal and vertical photographs (colored rectangle aspect ratio, 0.548 ; photograph aspect ratio, 0.676 ).

\section{Procedure}

The slides were presented on a wide screen in a large darkened auditorium with a seating capacity of 435 . The height and width of each projection was $8.0 \times 5.4 \mathrm{ft}$ in Part 1 and $11.0 \times 7.4 \mathrm{ft}$ in Part 2. Subjects sat between 18 and $80 \mathrm{ft}$ from the screen, with the majority between 25 and $65 \mathrm{ft}$ away.

Part 1. The subjects were exposed to each pair of slides for $5 \mathrm{sec}$, and there was a 5-sec interval between slides. After each pair was viewed, the subjects indicated on a form which slide they preferred: left, right, or neither.

Part 2. The subjects were exposed to single slides for $5 \mathrm{sec}$ each, with a 5-sec interval. After each slide, the subjects rated, on a scale of 0 to 10 ( 0 , least relaxed; 5 , neutral; 10 , most relaxed), how relaxed they had felt looking at the slide.

\section{RESULTS}

In Part 1 , there were no significant preferences for either vertical or horizontal rectangles in any of the 10 relevant comparisons. Nevertheless, the responses of the vast majority of the subjects did denote a preference: in all 10 of the comparisons, an average of only $21 \%$ chose "no preference" (the high was $26.8 \%$ and the low, $15 \%$ ).
The lack of a generalized preference for vertical or horizontal orientation is made evident in the highly consistent and nearly even proportions of subjects who indicated in each presentation that they preferred one orientation over the other. Overall, vertical presentations were preferred $39 \%$ of the time, while horizontal orientations were preferred $40 \%$ of the time. The highest denoted preference never exceeded $47 \%$ and never fell below $33 \%$. Furthermore, there was no significant relationship between vertical/horizontal preference and sex, ethnic origin, location of the viewer in the auditorium, or any of a variety of other demographic variables.

In Part 2, there was also no significant relationship between orientation of rectangle and subjective reports of relaxation.

\section{SUMMARY}

The results suggest that there may be no preference for horizontally dominant versus vertically dominant rectangles, at least with respect to the proportions, types, colors, sizes, and viewing distances used in this study. A comparison of responses to rectangles that are alike in every way except for orientation fails to elicit any indication of a predisposition to one orientation over the other.

In future research, we will employ nonidentical stimuli to explore further whether there are preferences for rectangular images of different orientations. We have also begun experimenting with vertically dominant television images, and will be measuring viewer response to these as well.

\section{REFERENCES}

Hintz, J. M., \& Nelson, T. M. (1970). Golden section: Reassessment of the perimetric hypothesis. American Journal of Psychology, 83, 126-129.

KRUGMAN, H. (1971). Brain wave measures of media involvement. Journal of Advertising Research, 11, 3-9.

Mulholland, T. (1974). Training visual attention. Academic Therapy, 10, 5-17.

Plug, C. (1980). The golden section hypothesis. American Journal of Psychology, 93, 467-487.

Schiffman, H. R. (1966). Golden section: Preferred figural orientation. Perception \& Psychophysics, 1, 193-194.

SCHIFFMAN, H. R. (1969). Figural preference and the visual field. Perception \& Psychophysics, 6, 92-94.

SCHIFFMAN, H. R., \& BoBKo, D. J. (1978). Preference in linear proportioning: The golden section reexamined. Perception \& Psychophysics, 24, 102-103.

(Manuscript received October 3, 1988.) 